

\title{
EFEITOS DA INCORPORAÇÃO DE JULGAMENTOS NA AVALIAÇÃO DA EFICIÊNCIA DE CLUBES DE FUTEBOL: UMA ABORDAGEM POR DATA ENVELOPMENT ANALYSIS
}

\author{
EFFECTS OF INCORPORATING JUDGMENTS IN FOOTBALL CLUB EFFICIENCY \\ ASSESSMENT: A DATA ENVELOPMENT ANALYSIS APPROACH
}

\author{
iD Alexandre de Cássio Rodrigues ${ }^{1}$ \\ iD Tiago Silveira Gontijo ${ }^{2}$ \\ iD Carlos Alberto Gonçalves ${ }^{3}$ \\ Thiago Henrique Martins Pereira ${ }^{4}$
}

\author{
${ }^{1}$ Doutor em Administração \\ Universidade FUMEC. \\ Belo Horizonte, MG - Brasil. \\ alexandrerodrigues.engprod@gmail.com \\ ${ }^{2}$ Mestre em Engenharia de Produção \\ Universidade Federal de Minas Gerais - UFMG. \\ Belo Horizonte, MG - Brasil. \\ tsgontijo@hotmail.com \\ ${ }^{3}$ Doutorado em Administração \\ Universidade Federal de Minas Gerais - UFMG. \\ Belo Horizonte, MG - Brasil. \\ carlos@face.ufmg.br \\ ${ }^{4}$ Doutor em Administração \\ Universidade FUMEC. \\ Belo Horizonte, MG - Brasil. \\ tigoemtv@hotmail.com
}

Recebido em: 27 jun. 2020 Aprovado em: 08 ago. 2020
Resumo: O objetivo deste artigo é apresentar um modelo DEA que incorpore julgamentos sobre a importância relativa de inputs e outputs na avaliação da eficiência dos clubes de futebol. Assim, aplicou-se esta metodologia para avaliar a eficiência dos 20 clubes que, em 2016, participaram do Campeonato Brasileiro de Futebol da Série A. Os resultados revelaram que, enquanto o modelo DEA clássico identificou nove clubes eficientes, o modelo proposto classificou apenas cinco. Apurou-se ainda que os clubes com maiores ativos tenderam a ser menos eficientes e que o tamanho da torcida presente nos estádios ou o histórico do desempenho esportivo não afetaram a eficiência daquelas organizações. Concluiu-se que os modelos clássicos de DEA, por ignorarem os julgamentos sobre a importância dos inputs e outputs, superestimam os escores de eficiência dos clubes e que o modelo proposto, ao impedir que pesos baixos sejam atribuídos a variáveis importantes, discrimina melhor os clubes eficientes.

Palavras-chave: Eficiência. Data Envelopment Analysis. Julgamentos. Clubes de futebol.

Abstract: The purpose of this paper is to present a DEA model that incorporates judgments about the importance of inputs and outputs in evaluating the effectiveness of soccer clubs. In the modeling the judgments on the importance of the operational expenses (input) and operational revenues and sports performance (outputs) are incorporated to a classic DEA model. So, this model is applied to evaluate the efficiency of the 20 clubs that participated in the Brazilian Serie A Football Championship in 2016. In addition, it is verified that contextual factors influenced that efficiency. The results revealed that while the classic DEA model identified nine efficient clubs, the proposed model ranked only five. It was also found that clubs with greater assets tended to be less efficient and that the size of the fans present in the stadiums or the history of sports performance did not affect the efficiency of those organizations. It was concluded that the classic DEA models, by ignoring the judgments about the importance of inputs and outputs, overestimate the efficiency scores of clubs and that the proposed model, by preventing low weights from being attributed to important variables, better discriminates efficient clubs.

Keywords: Efficiency. Data Envelopment Analysis. Judgments. Football clubs. 


\section{Introdução}

O futebol é o esporte mais popular do Brasil e é tido como um objeto fortemente atrelado a cultura nacional. Além de sua relevância local, destaca-se que o futebol representa uma importante fonte de divisas para as agremiações esportivas nacionais, constituindo assim, um produto de exportação tipicamente brasileiro. Em função de sua relevância econômica e social, a eficiência das equipes de futebol do Brasil é um objeto de interesse de diferentes players.

Estudos sobre a mensuração da eficiência de organizações são amplamente debatidos pela literatura nacional (Camioto, Rebelatto \& Rocha, 2016; Périco, Santana \& Rebelatto, 2017; Ferraz et al. 2018) e internacional (Zhou et al. 2018; Rashidi \& Cullinane, 2019; Tran et al., 2019), uma vez que, em um contexto de copetição global e de recursos escassos (Glauner, 2018), a performance de uma dada instituição pode ser considerada como um elemento de diferenciação e de até mesmo de sobrevivência (Shih \& Putri, 2018).

Especificamente no tocante ao mercado desportivo, destaca-se que o desempenho de clubes de futebol desperta o interesse dos torcedores, das empresas patrocinadoras e da mídia. No entanto, avaliar, de forma sistêmica, o desempenho dessas organizações não é uma tarefa simples (Plumley, Wilson \& Ramchandani, 2014), pois os clubes de futebol precisam atingir, simultaneamente, tanto o êxito esportivo quanto o financeiro (Guzmán, 2006; Pestana Barros, Assaf \& Sá-Earp, 2010). Afinal, idealmente, os recursos investidos em uma equipe altamente competitiva deveriam resultar em maior probabilidade de sucesso esportivo e, consequentemente, em aumento de receitas, as quais poderiam ser reinvestidas na contratação de bons jogadores, gerando, assim, um ciclo virtuoso (Espitia-Escuer \& García-Cebrián, 2010; Kern, Schwarzmann \& Wiedenegger, 2012).

Em pesquisas acadêmicas que visam avaliar a eficiência de clubes de futebol, isto é, a habilidade destas entidades em obter máximos produtos, dados os insumos à disposição (Zhu, 2014), frequentemente, têm-se feito o uso de modelos clássicos de Análise Envoltória de Dados (Data Envelopment Analysis - DEA) (Plumley, Wilson \& Ramchandani, 2014). A DEA compara o desempenho de unidades operacionais similares (Decision Making Units - DMUs), que consomem os mesmos inputs para produzir os mesmos outputs, diferenciando-se apenas nas quantidades consumidas e produzidas (Emouznejad \& Yang, 2017).

Uma particularidade dos modelos clássicos de DEA é a atribuição de pesos aos inputs e outputs de acordo com a melhor contribuição que estes possam oferecer (Charnes, Cooper \& Rhodes, 1978; Banker, Charnes \& Cooper, 1984). Esta flexibilidade faz com que pesos baixos sejam atribuídos às variáveis nas quais as DMUs tenham pior desempenho, mesmo que estas sejam importantes no julgamento de especialistas da área (Färe et al., 2015; Liu, Sharp \& Wu, 2006; Thanassoulis, Portela \& Allen, 2004). Logo, os modelos clássicos de DEA podem não ser apropriados para avaliar a eficiência de 
clubes de futebol, já que, por exemplo, podem ser classificados como eficientes clubes que tenham obtido bom desempenho esportivo, ainda que o desempenho financeiro deles tenha sido pífio (Pyatunin et al., 2016).

Uma revisão dos métodos de restrições aos pesos é apresentada por Joro e Korhonen (2015) e aplicações recentes desta abordagem na área de esportes incluem a avaliação da eficiência de países participantes de jogos olímpicos (Li et al., 2015), em que, nesta ordem, é dada maior importância às medalhas de ouro, prata e bronze, e de clubes de futebol (Hamidi, Sajadi \& Soleimani-Damaneh, 2011), em que se admite que a pontuação conquistada no campeonato seja mais importante que a receita obtida na temporada, que, por sua vez, é mais importante que a quantidade de torcedores presente nos estádios.

Apesar dos modeloes de DEA com restrições aos pesos serem tradicionais em estudos correlatos ao esporte, sobretudo em análises sobre a eficiência nas copas do mundo de futebol (Arabzad, Berjis \& Shirouyehzad, 2018), no futebol europeu (Espitia Escuer \& García Cebrián, 2019), em jogos olímpicos (Rodrigues, Gonçalves \& Gontijo, 2019) e em jogos panamericanos (Valério \& AnguloMeza, 2013), há a necessidade de pesquisas específicas sobre o desempenho dos clubes de futebol do Brasil.

Diante disso, formula-se a seguinte questão de pesquisa: quais são os fatores determinantes da eficiência (ou da ineficiência) dos clubes de futebol brasileiro da série A? De modo a responder a esta pergunta, o objetivo geral deste artigo é apresentar um modelo de DEA que na avaliação da eficiência de clubes de futebol incorpora julgamentos sobre a importância dos inputs e outputs baseados. Tal incorporação foi feita por meio da introdução de restrições aos pesos atribuídos àquelas variáveis pelos modelos de DEA clássicos. Em seguida, utilizou-se o modelo proposto para avaliar a eficiência dos clubes que, em 2016, participaram do Campeonato Brasileiro de Futebol - Série A. Adicionalmente, verificouse quais foram os fatores contextuais que influenciaram a eficiência daquelas organizações. Avaliar a eficiência de clubes de futebol do Brasil é relevante porque recentemente houveram mudanças na legislação que obrigaram estas entidades a adotarem práticas de responsabilidade fiscal e financeira e de gestão transparente e democrática (Brasil, 2015; Marotz, Marquezan \& Diehl, 2020), que se somaram às necessidades de ampla divulgação das demonstrações contábeis (Brasil, 2003) e de estruturação dos clubes de futebol em bases empresariais (Brasil, 1998). Além disso, sob a perspectiva teórica, ao se utilizarem os modelos de DEA com julgamentos sobre a importância dos inputs e outputs, ampliam-se os debates feitos em Freitas, Farias \& Flach (2017), Dantas, Machado \& Macedo (2015), Pereira et al. (2015), Nascimento et at. (2015) e Dantas \& Boente (2012), que ao avaliarem a eficiência de clubes brasileiros de futebol utilizaram modelos clássicos de DEA, os quais, conforme supracitado, podem gerar resultados equivocados. 
Por fim, destaca-se que o presente artigo contribui para a popularização da Pesquisa Operacional, uma vez que este trabalho reitera a importância das contribuições dos métodos de otimização para o planejamento e execução de instrumentos de aferição da performance de organizações esportivas. Desta forma, amplia-se o rol de aplicações e áreas de trabalho capazes de serem preenchidas por Engenheiros(as) de Produção. Especificamente no que diz respeito aos clubes de futebol do Brasil, este artigo poderá ser importante, uma vez que apresenta maneiras acuradas de se medir o desempenho destas entidades, bem como aborda quais são os fatores que relacionam-se com o desempenho esportivo, constituindo assim, importante arcabouço para o planejamento e a modernização do esporte mais popular do Brasil.

Além desta introdução, este artigo contém mais quatro sessões. A segunda apresenta um modelo de DEA que incorporou os julgamentos na avaliação da eficiência de clubes de futebol. $\mathrm{Na}$ terceira são detalhados os métodos de pesquisa; a seguir discutem-se os resultados obtidos e, por fim, têm-se as considerações finais.

\section{Um modelo de DEA que incorpora julgamentos na avaliação da eficiência de clubes de futebol}

Conceitualmente, tem-se que a eficiência constitui um conceito relativo e que compara aquilo que foi produzido, dados os recursos disponíveis, com tudo aquilo que poderia ser gerado com os mesmos recursos (Zhu, 2014). Desta forma, considera-se como eficiente aquela firma que, em comparação com as outras, alcança níveis máximos outputs a partir de um dado conjunto de inputs (orientação a outputs) ou que utiliza menos inputs para gerar uma certa quantidade de outputs (orientação a inputs)

A DEA é uma técnica não paramétrica de mensuração de eficiência, a qual foi difundida sobretudo a partir dos trabalhos seminais de Charnes, Cooper \& Rhodes (1978) (modelo CCR) e Banker, Charnes \& Cooper (1984) (modelo BCC). A principal diferença entre estes modelos clássicos é que enquanto o primeiro pressupõe retornos constantes de escala, isto é, que qualquer variação nos inputs implique em variação proporcional nos outputs, o segundo contempla o pressuposto de retornos variáveis de escala, ou seja, que para determinados volumes de inputs, a variação de outputs perca a proporcionalidade.

No tocante a formulação matemática proposta aos modelos de DEA, conforme Bogetoft \& Otto (2010), assume-se que "N unidades tomadoras de decisão" (Decision Making Units - DMUs) adotem uma mesma tecnologia de produção para modificar m inputs $x_{n}=\left(x_{n 1}, \ldots, x_{n m}\right) \in \mathfrak{R}_{+}^{m}$ em s outputs $y_{n}=\left(y_{n 1}, \ldots, y_{n s}\right) \in \mathfrak{R}_{+}^{s}$. Desta forma, o escore de eficiência da DMUo (objetivo), $\theta_{o}$, é representado pela equação (1): 


$$
\theta_{o}=\frac{\sum_{j=1}^{s} u_{j} y_{o j}}{\sum_{i=1}^{m} y_{i} x_{o i}}
$$

onde $v_{i}(\mathrm{i}=1, \ldots, \mathrm{m})$ e $\boldsymbol{u}_{j}(\mathrm{j}=1, \ldots, \mathrm{s})$ indicam, respectivamente, os pesos que o modelo confere aos inputs e outputs da $\mathrm{DMU}_{0}$. Esta atribuição é feita de maneira a maximar a eficiência da $\mathrm{DMU}_{0}$, que Ihe é mais favorável, e é restrita ao fato de que os pesos aplicados às demais DMUs não podem gerar escores de eficiência superior à unidade. De modo que não existam infinitas soluções para o problema, no caso de modelos com orientação a outputs e sob o pressuposto de retornos variáveis de escala, os quais serão utilizados neste trabalho, torna-se o numerador de (1) igual a uma constante, normalmente um, o que faz com que os escores de eficiência variem entre zero e um. Dessa forma, obtêm-se o modelo de multiplicadores (Zhu, 2014, p.50), o qual é mostrado na equação (2):

$$
\begin{gathered}
\theta_{o}=\operatorname{Max}_{v, u} \frac{1}{\sum_{i=1}^{\infty} y_{i} x_{o i}}+v_{o}=M_{v, u} i n \sum_{i=1} y_{i} x_{o i}+v_{o} \\
\sum_{j=1}^{\infty} u_{j} y_{n j}-\sum_{i=1}^{m} y_{i} x_{n i} \leq 0 \\
v_{i}, u_{j} \geq 0, \forall i, j
\end{gathered}
$$

Representa-se a solução ótima de (2) por $\left(\theta^{*} \mu^{*}, v^{*}\right)$. Assim, temos que a DMU。 será eficiente, se e somente se, $\theta^{*}=1$ e todos os valores de $u^{*}$ e $v^{*}$ forem positivos. Em caso adverso, ou seja, se

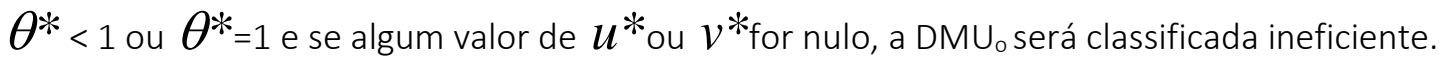

Em acréscimo, destaca-se os modelos de DEA clássicos atribuem pesos aos inputs e outputs de modo a maximizar os escores de eficiência das DMUs. Por um lado, isso permite identificar DMUs ineficientes, as quais apresentam baixo desempenho, mesmo após escolherem os pesos que lhe são mais favoráveis. Por outro, tal flexibilidade ignora quaisquer julgamentos dos especialistas em relação à importância dos inputs e outputs, uma vez que podem ser atribuídos pesos maiores a variáveis pouco relevantes ou pesos nulos a outras consideradas primordiais (Joro \& Korhonen, 2015). Nesse sentido, há que se destacar que julgamentos englobam sobre visões prévias em relação aos inputs e outputs, da relação entre alguns inputs e outputs, de DMUs eficientes e ineficientes e das substituições de inputs/outputs (Allen et al., 1997). Esses julgamentos podem ser incorporados aos modelos clássicos de DEA por meio de restrições aos pesos atribuídos aos inputs e/ou outputs (Joro \& Korhonen, 2015). 
Diante do exposto, na modelagem proposta neste trabalho admite-se que $\mathrm{N}$ clubes de futebol sejam as DMUs. No entanto, há que se frisar que, como ilustrado no Quadro 1, na avaliação de eficiência de clubes de futebol não há um consenso sobre quais inputs e outputs devam ser utilizados. Apesar disso, observa-se que, em geral, os inputs estão associados às despesas realizadas enquanto que os outputs se relacionam ao desempenho financeiro, dado pelas receitas obtidas, e ao desempenho esportivo, mensurado pela pontuação conquistada em um determinado campeonato. Sendo assim, essas serão as variáveis consideradas no modelo. Destaca-se que apesar de relevantes e de terem utilizado importantes variáveis correlatas aos clubes de futebol do Brasil, os trabalhos apresentados no Quadro 1 utilziaram os modelos clássicos de DEA (CCR e BCC), ou seja, desconsideraram o efeito da importância relativa dos inputs e dos outputs do modelo DEA no cômputo da eficiência das equipes.

\section{Quadro 1}

Inputs e outputs utilizados na avaliação da eficiência de clubes de futebol

\begin{tabular}{|c|c|c|}
\hline Trabalho & Inputs & Outputs \\
\hline $\begin{array}{l}\text { Dantes \& Boente } \\
\text { (2013) }\end{array}$ & $\begin{array}{l}\text { 1. Custos com atividades de futebol } \\
\text { 2. Ativo total }\end{array}$ & $\begin{array}{l}\text { 1. Receitas com atividades de } \\
\text { futebol } \\
\text { 2. Aproveitamento de pontos } \\
\text { 3. Número de títulos conquistados }\end{array}$ \\
\hline $\begin{array}{l}\text { Nascimento et al. } \\
(2015)\end{array}$ & $\begin{array}{l}\text { 1. Ativo imobilizado } \\
\text { 2. Intangível } \\
\text { 3. Custos com pessoal } \\
\text { 4. Despesas gerais }\end{array}$ & $\begin{array}{l}\text { 1. Pontos conquistados no ranking } \\
\text { da CBF }\end{array}$ \\
\hline Pereira et al. (2015) & $\begin{array}{l}\text { 1. Custo do departamento de } \\
\text { futebol } \\
\text { 2. Ativo total }\end{array}$ & $\begin{array}{l}\text { 1. Receita total } \\
\text { 2. Pontuação no ranking da CBF } \\
\text { 3. Valor de mercado }\end{array}$ \\
\hline $\begin{array}{l}\text { Dantas, Machado \& } \\
\text { Macedo (2015) }\end{array}$ & 1. Despesas operacionais & $\begin{array}{l}\text { 1. Receita operacional } \\
\text { 2. Pontuação do ranking da CBF }\end{array}$ \\
\hline $\begin{array}{l}\text { Freitas, Farias \& } \\
\text { Flach (2017) }\end{array}$ & $\begin{array}{l}\text { 1. Ativo total } \\
\text { 2. Despesas com pessoal }\end{array}$ & Receita total \\
\hline
\end{tabular}

Fonte: Elaborado pelos autores com base em: Dantas \& Boente (2012), Nascimento et at. (2015), Pereira et al. (2015), Dantas, Machado \& Macedo (2015) e Freitas \& Farias (2017).

Conforme já mencionado, o modelo proposto admite retornos variáveis de escala. Isso se justifica porque a adoção dos modelos de DEA com retornos constantes de escala requer que as DMUs estejam operando em escala ótima e "competição imperfeita, restrições financeiras, dentre outras causas, podem levar uma DMU a não operar em escala de produção ótima" (Ferreira \& Gomes, 2009, p. 194). Utiliza-se, ainda, a orientação a outputs, assim como fizeram Freitas, Farias \& Flach (2017), Dantas, Machado \& Macedo (2015), Pereira et al. (2015), Nascimento et at. (2015) e Dantas e Boente (2012). Logo, serão classificados como eficientes os clubes de futebol que, considerando as despesas realizadas, conseguiram maximizar os desempenhos financeiro e esportivo. Tais escolhas estão sumarizadas no seguinte modelo de DEA clássico, conforme é apresentado na equação (3): 


$$
\begin{gathered}
\theta_{o}=\operatorname{Min}_{v, u} v_{1} x_{o 1}+v_{o} \\
\sum_{j=1}^{2} u_{j} y_{n j}-v_{1} x_{n 1} \leq 0 \\
v_{i}, u_{j} \geq 0, \forall i, j
\end{gathered}
$$

Deve-se notar que o modelo dado pela equação (3) não incorpora julgamentos sobre a importância dos outputs e do input. No entanto, é razoável admitir que na avaliação da eficiência dos clubes de futebol, as despesas operacionais tenham maior importância que os desempenhos financeiro e esportivo, isto é, que $v_{1} \geq u_{1}$ e $v_{1} \geq u_{2}$, pois como bem observaram Barajas, Férnandez-Jardon \& Crolley (2005), se por um lado as receitas obtidas e a pontuação conquistada em determinado campeonato não podem ser controladas pelos clubes, por outro, as despesas podem sê-lo. Por sinal, recente relatório divulgado pelo Banco Itaú, que analisa a situação econômico-financeira dos clubes brasileiros de futebol, aponta que o controle de gastos é um fator crucial para se garantir a sustentabilidade daquelas organizações (Itaú, 2019). Além disso, ainda podem ser incorporados ao modelo de DEA clássico dado por (3), os julgamentos de que o desempenho financeiro em relação ao desempenho esportivo seja mais importante $\left(\mathrm{u}_{1} \geq \mathrm{u}_{2}\right)$, menos importante $\left(\mathrm{u}_{1} \leq \mathrm{u}_{2}\right)$ ou que tenha igual importância $\left(\mathrm{u}_{1}=\mathrm{u}_{2}\right)$. Este modelo foi aplicado na avaliação da eficiência de clubes brasileiros de futebol. Os métodos utilizados nesta pesquisa são apresentados a seguir. Por fim, destaca-se que o presente método é sensível quanto à escolha da importância relativa dos inputs e outputs escolhidos, entretando este problema foi mitigado neste artigo, uma vez que a preferência nas escolhas priorizou apenas os aspectos que são gerenciáveis pelos clubes de futebol.

\section{Métodos}

Neste trabalho, por meio de uma pesquisa quantitativa e descritiva, avaliou-se a eficiência de clubes brasileiros de futebol em 2016. A amostra, não-probabilística e intencional, foi constituída pelos 20 clubes que, naquele ano, participaram da principal competição nacional - o Campeonato Brasileiro de Futebol Série A: América-MG, Atlético-MG, Atlético-PR, Botafogo, Chapecoense, Corinthians, Coritiba, Cruzeiro, Figueirense, Flamengo, Fluminense, Grêmio, Internacional, Palmeiras, Ponte Preta, Santa Cruz, São Paulo, Sport e Vitória. Conforme apresentado na seção anterior, o input foram as despesas operacionais e outputs as receitas obtidas e a pontuação conquistada no campeonato. As variáveis financeiras foram obtidas a partir da consulta aos balanços patrimoniais dos clubes, os quais são divulgados em seus respectivos sítios na internet, enquanto que a pontuação conquistada no 
campeonato estava disponível no sítio da Confederação Brasileira de Futebol (CBF). As estatísticas descritivas dessas variáveis são mostradas na Tabela 1.

Tabela 1

Estatísticas descritivas do input e dos outputs

\begin{tabular}{lllll}
\multicolumn{1}{c}{ Variável } & \multicolumn{1}{c}{ Mínimo } & \multicolumn{1}{c}{ Máximo } & \multicolumn{1}{c}{ Média } & \multicolumn{1}{c}{ Desvio-padrão } \\
Despesas operacionais (R\$) & $36.854 .071,92$ & $470.911 .736,00$ & $185.620 .590,20$ & $130.099 .041,83$ \\
Receitas operacionais (R\$) & $36.854 .071,92$ & $510.074 .00,00$ & $211.186 .040,20$ & $144.148 .998,48$ \\
Pontos & 28 & 80 & 52,15 & 12,83
\end{tabular}

Fonte: Elaborada pelos autores a partir dos dados da pesquisa.

Para fins de comparação, avaliou-se a eficiência dos clubes brasileiros de futebol por meio dos modelos DEA clássicos e com a incorporação de julgamentos sobre a importância do input e dos outputs. Nesta etapa foi utilizado o software Sistema Integrado de Apoio à Decisão (SIAD), que trata-se de uma ferramenta gratuita respaldada pela comunidade acadêmica de Data Envelopment Analysis (Meza et al., 2005).

Há que se frisar que apesar de a DEA calcular os escores de eficiência, essa técnica não identifica os fatores que os afetam. Por isso, para fazê-lo, conforme recomendado por McDonald (2009), utilizouse um modelo de regressão Tobit, o qual se justifica porque a variável dependente, o escore de eficiência calculado pela DEA $(\theta)$, é limitada à unidade, conforme é apresentado na equação (4):

$$
\theta_{n}=\left\{\begin{array}{l}
\theta_{n}^{*}, \text { if } 0<\theta_{n}^{*}<1 \\
0, \text { if } \theta_{n}^{*}<0 \\
1, \text { if } \theta_{n}^{*} \geq 1
\end{array}\right.
$$

Nesse contexto, analisou-se se os escores de eficiência eram afetados pelo ativo (ativo), pelo tamanho médio da torcida presente nos estádios (torcida) e pela pontuação do clube no ranking da CBF, variáveis que no curto prazo não podem ser controlados pelas gestores. Além disso, por meio de uma variável dummy, esses efeitos foram controlados pelo fato de o clube ter vencido ou não o campeonato estadual em 2016. Na Tabela 2 as estatísticas referentes à assimetria e à curtose mostram que as variáveis independentes contínuas eram assimétricas e apresentavam pesos nas caudas (ativo, principalmente). 
Tabela 2

Estatísticas descritivas das variáveis contínuas do modelo de regressão Tobit

\begin{tabular}{llllll}
$\quad$ Variável & \multicolumn{1}{c}{ Média } & \multicolumn{1}{c}{ Mediana } & Desvio-padrão & \multicolumn{1}{c}{ Assimetria } & \multicolumn{1}{c}{ Curtose } \\
ativo (R\$) & $471.840 .107,70$ & $287.049 .264,00$ & $498.126 .876,00$ & 2,258 & 6,453 \\
torcida & $15.180,20$ & $12.982,00$ & $8.487,71$ & 0,513 & $-0,674$ \\
rank_CBF & $10.815,40$ & $11.950,00$ & $3.281,12$ & $-0,674$ & $-0,903$
\end{tabular}

Fonte: Elaborada pelos autores a partir dos dados da pesquisa.

Para atenuar esse problema, conforme sugerido por Green (2008), tomou-se o logaritmo de todas as variáveis contínuas, originando o modelo dado pela equação (5). Desse modo, os coeficientes estimados forneceram a elasticidade, isto é, a variação percentual dos escores de eficiência correspondente à variação percentual dos fatores explicativos, conforme indicado na equação (5):

$$
\ln \left(\theta_{n}\right)=\beta_{0}+\beta_{1} \ln \left(\text { ativ } \phi_{n}+\beta_{2} \ln (\text { torcid }) a+\beta_{3} \ln (\operatorname{rank} C B F)_{n}+\beta_{4} \text { estaduat } \varepsilon_{i}\right.
$$

O software Gretl foi utilizado para estimar os coeficientes do modelo de regressão, uma vez que trata-se de uma ferramenta gratuita e respaldada pela literatura internacional sobre econometria (Mixon Jr \& Smith, 2006). Os resultados encontrados são apresentados e discutidos na próxima seção.

\section{Resultados e discussões}

\subsection{Escores de eficiência dos clubes de futebol - modelo de DEA clássico}

Os escores de eficiência dos 20 clubes que participaram do Campeonato Brasileiro de Futebol em 2016 são mostrados na Tabela 3. Os valores foram calculados utilizando-se um modelo de DEA clássico, orientado a outputs, sob o pressuposto de retornos variáveis de escala e sem incorporar julgamentos sobre a importância dada às despesas, às receitas operacionais e à pontuação obtida no campeonato, ou seja, fez-se uso de procedimentos similares aos adotados por Freitas \& Farias (2017), Pereira et al. (2015), Nascimento et at. (2015) e Dantas \& Boente (2013). Também são destacados os pesos atribuídos ao input e aos outputs e, na última linha da tabela, são expostos, respectivamente, o número de clubes de futebol classificados como eficientes e que, para cada variável, o modelo de DEA clássico atribuiu peso não-nulo. 


\section{Tabela 3}

Eficiência e pesos atribuídos ao input e aos outputs - modelo de DEA clássico

\begin{tabular}{|c|c|c|c|c|c|}
\hline \multirow{2}{*}{$\mathrm{N}$} & \multirow{2}{*}{ Clube } & \multirow{2}{*}{$\theta$} & \multicolumn{3}{|c|}{ Pesos } \\
\hline & & & Despesas & Receitas & Pontos \\
\hline 1 & Santa Cruz & 1,00 & 0,00000007 & 0,00000003 & 0,00000000 \\
\hline 2 & Chapecoense & 1,00 & 0,00000002 & 0,00000001 & 0,00450981 \\
\hline 3 & Internacional & 1,00 & 0,00000000 & 0,00000000 & 0,02032216 \\
\hline 4 & Ponte Preta & 1,00 & 0,00000000 & 0,00000000 & 0,01988462 \\
\hline 5 & Flamengo & 1,00 & 0,00000000 & 0,00000000 & 0,01153106 \\
\hline 6 & Santos & 1,00 & 0,00000000 & 0,00000000 & 0,00842103 \\
\hline 7 & Palmeiras & 1,00 & 0,00000000 & 0,00000000 & 0,00637360 \\
\hline 8 & São Paulo & 1,00 & 0,00000000 & 0,00000000 & 0,00503014 \\
\hline 9 & Corinthians & 1,00 & 0,00000000 & 0,00000000 & 0,00000000 \\
\hline 10 & Atlético-PR & 0,97 & 0,00000000 & 0,00000000 & 0,02426224 \\
\hline 11 & Botafogo & 0,97 & 0,00000000 & 0,00000000 & 0,02486788 \\
\hline 12 & Vitória & 0,91 & 0,00000002 & 0,00000001 & 0,00000000 \\
\hline 13 & Figueirense & 0,87 & 0,00000003 & 0,00000001 & 0,00000000 \\
\hline 14 & Atlético-MG & 0,86 & 0,00000000 & 0,00000000 & 0,01393731 \\
\hline 15 & América & 0,84 & 0,00000004 & 0,00000002 & 0,00000000 \\
\hline 16 & Coritiba & 0,77 & 0,00000000 & 0,00000000 & 0,02415226 \\
\hline 17 & Sport & 0,75 & 0,00000000 & 0,00000000 & 0,03245913 \\
\hline 18 & Grêmio & 0,74 & 0,00000000 & 0,00000000 & 0,03328711 \\
\hline 19 & Cruzeiro & 0,71 & 0,00000000 & 0,00000000 & 0,03957665 \\
\hline 20 & Fluminense & 0,67 & 0,00000000 & 0,00000000 & 0,00953237 \\
\hline Total & & 9 & 5 & 5 & 15 \\
\hline
\end{tabular}

Fonte: Elaborada pelos autores a partir dos resultados da pesquisa

Analisando-se os resultados na Tabela 3, observa-se que dos vinte clubes de futebol avaliados nove foram classificados como eficientes. Tem-se ainda que no cálculo dos escores de eficiência o modelo de DEA clássico atribuiu peso não-nulo às despesas e receitas operacionais de cinco clubes e que a pontuação obtida no campeonato foi levada em consideração na avaliação da eficiência de 15 clubes. Nesse sentido, nota-se que apenas a um clube (o Chapecoense, em destaque) foi atribuído peso não-nulo a todas as variáveis. Os valores desses pesos indicam que foi dada importância intermediária às despesas operacionais, menor importância às receitas operacionais e maior importância à pontuação obtida no campeonato. Logo, tais resultados revelam o modelo de DEA clássico, na maioria dos casos, ignorou aquelas variáveis e, quando o fez, atribui importância incoerente com o julgamento de especialistas do setor.

\subsection{Escores de eficiência dos clubes de futebol - modelo de DEA com julgamentos}

A Tabela 4 mostra os escores de eficiência 20 clubes que participaram do Campeonato Brasileiro de Futebol em 2016, os quais foram calculados utilizando-se um modelo de DEA clássico, orientado a 
outputs, sob o pressuposto de retornos variáveis de escala e com a incorporação de julgamentos sobre a importância das despesas operacionais, das receitas operacionais e da pontuação obtida no campeonato. Como feito anteriormente, são destacados os pesos atribuídos àquelas variáveis e expostos o número de clubes de futebol classificados como eficientes e que o modelo DEA proposto atribuiu, para cada input e output, peso não-nulo. Destaca-se que para evitar os efeitos das diferentes ordens de grandeza entre as variáveis, os pesos foram normalizados (Roll, Cook \& Golany, 1991).

Tabela 4

Eficiência e pesos - modelo de DEA com incorporação de julgamentos

\begin{tabular}{|c|c|c|c|c|c|c|c|c|c|c|c|c|c|}
\hline \multirow{3}{*}{$\mathrm{N}$} & \multirow{3}{*}{ Clube } & \multicolumn{4}{|c|}{ Financeiro } & \multicolumn{4}{|c|}{ Esportivo } & \multicolumn{4}{|c|}{ Financeiro-Esportivo } \\
\hline & & \multirow{2}{*}{$\theta$} & \multicolumn{3}{|c|}{ Pesos } & \multirow{2}{*}{$\theta$} & \multicolumn{3}{|c|}{ Pesos } & \multirow{2}{*}{$\theta$} & \multicolumn{3}{|c|}{ Pesos } \\
\hline & & & Desp & $\operatorname{Rec}$ & Pontos & & Desp & $\operatorname{Rec}$ & Pontos & & Desp & $\operatorname{Rec}$ & Pontos \\
\hline 1 & Santa Cruz & 1,00 & 23,55 & 2,18 & 2,18 & 1,00 & 21,77 & 0,00 & 2,58 & 1,00 & 23,55 & 2,18 & 2,18 \\
\hline 2 & Chapecoense & 1,00 & 13,59 & 1,26 & 1,26 & 1,00 & 1,73 & 0,00 & 1,54 & 1,00 & 13,59 & 1,26 & 1,26 \\
\hline 3 & Santos & 1,00 & 1,26 & 0,72 & 0,72 & 1,00 & 2,02 & 0,72 & 0,72 & 1,00 & 1,26 & 0,72 & 0,72 \\
\hline 4 & Palmeiras & 1,00 & 1,02 & 0,61 & 0,51 & 1,00 & 0,97 & 0,55 & 0,55 & 1,00 & 0,76 & 0,55 & 0,55 \\
\hline 5 & Flamengo & 1,00 & 1,00 & 1,00 & 0,00 & 1,00 & 0,73 & 0,53 & 0,53 & 1,00 & 0,73 & 0,53 & 0,53 \\
\hline 6 & Ponte Preta & 0,91 & 3,55 & 1,27 & 1,27 & 0,97 & 1,70 & 0,00 & 1,51 & 0,91 & 3,55 & 1,27 & 1,27 \\
\hline 7 & Botafogo & 0,88 & 2,71 & 0,97 & 0,97 & 0,92 & 1,53 & 0,00 & 1,36 & 0,88 & 2,71 & 0,97 & 0,97 \\
\hline 8 & Atlético-PR & 0,87 & 2,92 & 1,04 & 1,04 & 0,92 & 1,58 & 0,00 & 1,40 & 0,87 & 2,92 & 1,04 & 1,04 \\
\hline 9 & Vitória & 0,91 & 7,62 & 4,56 & 0,00 & 0,82 & 3,58 & 1,28 & 1,28 & 0,82 & 3,58 & 1,28 & 1,28 \\
\hline 10 & Corinthians & 0,80 & 1,11 & 1,11 & 0,00 & 0,79 & 0,63 & 0,63 & 0,63 & 0,79 & 0,63 & 0,63 & 0,63 \\
\hline 11 & Coritiba & 0,74 & 3,54 & 1,27 & 1,27 & 0,76 & 1,96 & 0,00 & 1,74 & 0,74 & 3,54 & 1,27 & 1,27 \\
\hline 12 & Figueirense & 0,87 & 12,04 & 7,20 & 0,00 & 0,73 & 4,65 & 1,66 & 1,66 & 0,73 & 4,65 & 1,66 & 1,66 \\
\hline 13 & Atlético-MG & 0,73 & 0,72 & 0,72 & 0,72 & 0,73 & 0,76 & 0,67 & 0,76 & 0,73 & 0,72 & 0,72 & 0,72 \\
\hline 14 & Grêmio & 0,70 & 1,62 & 0,93 & 0,93 & 0,71 & 1,20 & 0,50 & 1,20 & 0,70 & 1,62 & 0,93 & 0,93 \\
\hline 15 & Sport & 0,70 & 3,33 & 1,19 & 1,19 & 0,72 & 1,92 & 0,00 & 1,70 & 0,70 & 3,33 & 1,19 & 1,19 \\
\hline 16 & São Paulo & 0,68 & 0,70 & 0,70 & 0,70 & 0,68 & 0,70 & 0,70 & 0,70 & 0,68 & 0,70 & 0,70 & 0,70 \\
\hline 17 & Fluminense & 0,67 & 1,52 & 0,91 & 0,76 & 0,66 & 1,14 & 0,83 & 0,83 & 0,66 & 1,14 & 0,83 & 0,83 \\
\hline 18 & Cruzeiro & 0,62 & 1,58 & 0,91 & 0,91 & 0,63 & 1,20 & 0,50 & 1,20 & 0,62 & 1,58 & 0,91 & 0,91 \\
\hline 19 & América & 0,84 & 20,49 & 8,57 & 0,00 & 0,61 & 23,20 & 2,14 & 2,14 & 0,61 & 23,20 & 2,14 & 2,14 \\
\hline 20 & Internacional & 0,41 & 1,07 & 1,07 & 1,07 & 0,41 & 1,13 & 1,00 & 1,13 & 0,41 & 1,07 & 1,07 & 1,07 \\
\hline & Total & 5 & 20 & 20 & 15 & 5 & 20 & 13 & 20 & 5 & 20 & 20 & 20 \\
\hline
\end{tabular}

Fonte: Elaborada pelos autores a partir dos resultados da pesquisa.

Os resultados mostrados na Tabela 4 relevam que nos três modelos apenas cinco clubes de futebol foram classificados como eficientes e que a variável admitida como sendo a mais importante despesas operacionais - foi incluída no cálculo dos escores de eficiência de todos os clubes. Percebe-se, ainda, que ao se calcular os escores de eficiência o modelo financeiro ignorou-se o desempenho esportivo de cinco clubes. Analogamente, no modelo esportivo, ignorou-se o desempenho financeiro de sete clubes. Contudo, no modelo financeiro-esportivo, todas as variáveis foram admitidas no 
cômputo dos escores de eficiência. Logo, por ser sistêmico, pode-se afirmar que o modelo financeiroesportivo é mais adequado para avaliar a eficiência de clubes de futebol que os demais.

Comparando-se os resultados nas Tabelas 3 e 4 o que se constata é que ao se incorporar julgamentos sobre a importância dada às despesas e receitas operacionais e à pontuação obtida no campeonato brasileiro, o número de clubes de futebol classificados como eficientes foi reduzido. Isso pode ser explicado pelo fato de que por meio daquela ação impediu-se que pesos baixos fossem atribuídos às variáveis consideradas importantes. Além disso, verifica-se que a incorporação dos julgamentos sobre a importância das variáveis gera escores de eficiência menores ou iguais àqueles calculados pelo modelo de DEA clássico. As estatísticas desses escores, expostas na Tabela 5, mostram que, de acordo com o modelo de DEA clássico, os clubes de futebol poderiam, em média, incrementar os outputs em até $10 \% \%(1-0,90)$, sem aumentar o nível de input. Analogamente, segundo o modelo de DEA com incorporação de julgamentos (financeiro-esportivo), os outputs poderiam ser incrementados em até $21 \%(1-0,79)$.

\section{Tabela 5}

Estatísticas descritivas dos escores de eficiência

\begin{tabular}{lcccc}
\multicolumn{1}{c}{ Escores de eficiência } & Mínimo & Máximo & Média & Desvio-padrão \\
DEA clássico & 0,67 & 1,00 & 0,90 & 0,12 \\
DEA financeiro-esportivo & 0,41 & 1,00 & 0,79 & 0,17
\end{tabular}

Fonte: Elaborada pelos autores a partir dos resultados da pesquisa

A fim de verificar se a diferença entre os escores de eficiência obtidos pelo modelo de DEA clássico e com incorporação de julgamentos era significativa foi realizado um teste t para amostras independentes. Os resultados encontrados são mostrados na Tabela 6:

\section{Tabela 6}

Resultados do teste t para amostras independentes - escores de eficiência

\begin{tabular}{|c|c|c|c|c|c|}
\hline & \multicolumn{2}{|c|}{$\begin{array}{c}\text { Teste Levene - igualdade de variâncias } \\
\text { Levene (1960) }\end{array}$} & \multicolumn{3}{|c|}{$\begin{array}{l}\text { Teste } \mathrm{t} \text { - igualdade de médias } \\
\text { Welch (1951) }\end{array}$} \\
\hline & Estatística F & P-value & Diferença & Estatística t & P-value \\
\hline Variâncias iguais & 2,21 & 0,15 & 0,12 & 2,46 & 0,02 \\
\hline Variâncias diferentes & & & 0,12 & 2,46 & 0,02 \\
\hline
\end{tabular}

Fonte: Elaborada pelos autores a partir dos resultados da pesquisa

Os resultados do teste de Leneve revelam, ao nível de 5\% significância, que a hipótese de igualdade de variâncias entre os escores de eficiência obtidos pelo modelo de DEA clássico e com 
incorporação de julgamentos não pode ser rejeitada. Assim, assumindo-se que as variâncias sejam iguais, rejeitou-se a hipótese de igualdade de média entre aqueles escores. Isso quer dizer que o modelo DEA clássico, ao ignorar os julgamentos da importância do input e dos outputs, de fato, superestimou os escores de eficiência dos clubes de futebol.

\subsection{Determinantes da eficiência dos clubes de futebol}

Para identificar quais são os fatores que afetaram a eficiência dos clubes de futebol, os escores calculados pelo modelo DEA que incorpora os julgamentos (modelo financeiro-esportivo) foram admitidos como a variável dependente de uma regressão Tobit. As variáveis dependentes foram: o ativo do clube (ativo), o tamanho médio da torcida presente nos estádios (torcida) e a pontuação do clube no ranking da CBF (rank_CBF). Considerou-se, ainda, a variável dummy estadual, que controla o fato de o clube ter vencido ou não o campeonato estadual em 2016.

Os resultados das estimações mostrados na Tabela 7 revelam que o conjunto de variáveis independentes consideradas explicaram satisfatoriamente a variação dos escores de eficiência dos clubes de futebol $\left(P>X^{2}=0,000\right)$, o que implica que o modelo apresentado é globalmente válido. Ademais, conforme resultados do teste Doornik-Hansen, a hipótese de normalidade dos resíduos não pôde ser rejeitada ao nível de significância de 10\%.

Tabela 7

Efeitos sobre a eficiência dos clubes de futebol

\begin{tabular}{lllll} 
& \multicolumn{1}{c}{ Coeficiente } & Erro-padrão & Estatística Z & P-value \\
Intercepto & 2,063 & 2,495 & 0,827 & 0,408 \\
In(ativo) & $-0,157$ & 0,081 & $-1,935$ & 0,053 \\
In(torcida) & 0,185 & 0,149 & 1,241 & 0,214 \\
In(rank_CBF) & $-0,103$ & 0,359 & $-0,290$ & 0,772 \\
estadual & $-0,005$ & 0,208 & $-0,022$ & 0,982 \\
\hline Estatística X & & & 21,688 & 0,000 \\
Teste Doornik-Hansen & & 0,524 & 0,769
\end{tabular}

Fonte: Elaborada pelos autores a partir dos resultados da pesquisa

Ainda com relação aos resultados expostos na Tabela 7, constata-se que, ao nível de $10 \%$ de significância, somente variável ativo apresentou influência sobre os escores de eficiência. O sinal negativo do coeficiente dessa variável sugere que clubes de futebol mais ricos tenderam a ser menos eficientes. De modo objetivo, uma variação positiva de $1 \%$ no ativo implicou na queda de $15,7 \%$ nos escores de eficiência dos clubes de futebol. Possivelmente, isso pode estar relacionado ao fato de clubes com maiores ativos, embora, em geral, contem com bons jogadores, cujos salários são muito altos, não 
necessariamente apresentam grandes receitas e bom desempenho no Campeonato Brasileiro de Futebol, simultaneamente.

Também se depreende dos resultados mostrados na Tabela 7 que o tamanho médio da torcida presente nos estádios e a pontuação dos clubes no ranking da CBF não afetaram a eficiência clubes brasileiros de futebol em 2016. Além disso, não foram verificadas diferenças significativas entre os escores de eficiência de clubes vencedores e não vencedores dos campeonatos de futebol em seus respectivos estados. O fato de o tamanho da torcida presente nos estádios não ter influenciado os escores de eficiência dos clubes não é surpreendente, pois Borges \& Petry (2016) constataram que o desempenho esportivo dos clubes não influência o comparecimento dos torcedores nas arenas de futebol. Certamente a maioria dos clubes avaliados deve possuir torcedores muito leais que, por exemplo, geram receita, independentemente da pontuação do time no campeonato. Todavia, os demais resultados contrariam os achados da literatura, pois Freitas, Farias \& Flach (2017) e Dantas, Machado \& Macedo (2015), respectivamente, concluíram que a pontuação dos clubes no ranking da CBF afeta negativamente os escores de eficiência e que clubes que conquistam títulos em determinada temporada tendem a ser mais eficientes. O input e os outputs, bem como a incorporação de julgamentos sobre a importância dessas variáveis, os quais afetam o valor dos escores de eficiência, são justificativas plausíveis para explicar tais divergências.

\section{Considerações finais}

O futebol é o esporte mais popular do Brasil, portanto, o desempenho gerencial dos clubes de futebol possui uma importância que extrapola os aspectos esportivos, perfazendo parcela relevante do produto interno bruto nacional. Assim, por se tratar de um objeto, tanto de entretenimento, quanto de exportação nacional, o desempenho dos clubes esportivos possui diversas externalidades positivas para a sociedade brasileira. Deste modo, analisar a performance destas entidades constitui importante exercício prático e teórico para a comunidade acadêmica e empresarial do Brasil.

Neste artigo apresentou-se um modelo de DEA que, ao avaliar a eficiência de clubes de futebol, incorporou julgamentos sobre a importância relativa dos inputs e outputs. Por serem controláveis pelos clubes, admitiu-se que o peso atribuído às despesas operacionais não fossem menores que aquele atribuído às despesas operacionais e que a pontuação conquistada em um determinado campeonato, os quais não podem ser controlados. Esses julgamentos foram incorporados a um modelo de DEA clássico por meio de restrições aos pesos atribuídos àquelas variáveis.

Ao se avaliar a eficiência dos clubes que, em 2016, participaram do Campeonato Brasileiro de Futebol Série A, verificou-se que o modelo proposto, ao não permitir que pesos baixos fossem atribuídos a variáveis consideradas importantes, discriminou melhor os clubes eficientes, quando comparado à 
classificação feita pelo modelo DEA clássico. Isso se deve ao fato de que os modelos clássicos, por ignorarem os julgamentos sobre a importância relativa dos inputs e dos outputs, podem superestimar os escores de eficiência dos clubes de futebol.

A principal contribuição teórica do modelo proposto neste trabalho é a possibilidade de impedir uma avalição equivocada em que clubes de futebol, a priori, ineficientes possam ser classificados como eficientes ao desconsiderem os julgamentos sobre a importância das variáveis. Do ponto de vista prático, a pesquisa contribui na medida em que evidencia que clubes de futebol, sobretudo os mais ricos, precisam ter a gestão aprimorada, pois obteve-se indícios de que quanto maior é o ativo, maior é a tendência que as despesas sejam realizadas de modo ineficiente.

Reconhece-se que os resultados aqui expostos não podem ser extrapolados, pois limitam-se aos clubes que, em 2016, participaram do Campeonato Brasileiro de Futebol. Além disso, salienta-se que como os escores de eficiência calculados pela DEA são diretamente influenciados pelos inputs e outputs selecionados, pelo tipo de retorno de escala, pela orientação do modelo e, no contexto desta pesquisa, pelo julgamento sobre a importância das variáveis, a adoção de novas escolhas podem gerar resultados diferentes. Por fim, como sugestão, recomenda-se que, em trabalhos futuros, o modelo desenvolvido nesta pesquisa seja utilizado para avaliar a eficiência de clubes de futebol de outros países ou campeontos e, ainda, de equipes de modalidades esportivas diferentes.

\section{Referências}

Allen, R., Athanassopoulos, A., Dyson, R. G., \& Thanassoulis, E. (1997). Weights restrictions and value judgements in data envelopment analysis: evolution, development and future directions. Annals of operations research, 73, 13-34. https://doi.org/10.1023/A:1018968909638

Arabzad, S. M., Berjis, N., \& Shirouyehzad, H. (2018). Ranking World Cup 2014 Football Matches by Data Envelopment Analysis Models with Common Weights. Iranian Journal of Optimization, 10(2), 139-150.

Banker, R. D., Charnes, A., \& Cooper, W. W. (1984). Some models for estimating technical and scale inefficiencies in data envelopment analysis. Management science, 30(9), 1078-1092. https://doi.org/10.1287/mnsc.30.9.1078

Barajas, A., Fernández-Jardón, C. M., \& Crolley, L. (2005). Does sports performance influence revenues and economic results in Spanish football? http://dx.doi.org/10.2139/ssrn.986365

Bogetoft, P., \& Otto, L. (2010). Benchmarking with Dea, Sfa, and R (Vol. 157). Springer Science \& Business Media. http://dx.doi.org/10.1007/978-1-4419-7961-2

Borges, G., \& Petry, J. (2016). O Impacto do Desempenho do Time na Presença de Torcedores no Estádio de Futebol. PODIUM Sport, Leisure and Tourism Review, 5(3), 60-74. 
Brasil. Lei n. 9.615, de 24 de março de 1998 (1998). Institui normas gerais sobre desporto e dá outras providências. Brasília/DF, Diário Oficial da União.

Brasil. Lei n. 10.672, de 15 de maio de 2003 (2003). Altera dispositivos da Lei no 9.615, de 24 de março de 1998, e dá outras providências. Brasília/DF, Diário Oficial da União.

Brasil. Lei n. 13.155, de 04 de agosto de 2015 (2015). Estabelece princípios e práticas de responsabilidade fiscal e financeira e de gestão transparente e democrática para entidades desportivas profissionais de futebol. Brasília/DF, Diário Oficial da União.

Camioto, F. D. C., Rebelatto, D. A. D. N., \& Rocha, R. T. (2016). Energy efficiency analysis of BRICS countries: a study using Data Envelopment Analysis. Gestão \& Produção, 23(1), 192-203. http://dx.doi.org/10.1590/0104-530X1567-13

Charnes, A., Cooper, W. W., \& Rhodes, E. (1978). Measuring the efficiency of decision making units. European journal of operational research, 2(6), 429-444. https://doi.org/10.1016/03772217(78)90138-8

Dantas, M. G., \& Boente, D. R. (2012). A utilização da análise envoltória de dados na medição de eficiência dos clubes brasileiros de futebol. Contabilidade Vista \& Revista, 23(2), 101-130.

Dantas, M. G., Machado, M. A. V., \& Macedo, M. A. (2015). Fatores determinantes da eficiência dos clubes de futebol do Brasil. Advances in Scientific and Applied Accounting, 8(1), 113-132. https://doi.org/10.14392/ASAA.2015080106

Rodrigues, A. C., Gonçalves, C. A., \& Gontijo, T. S. (2019). A two-stage DEA model to evaluate the efficiency of countries at the Rio 2016 Olympic Games". Economics Bulletin, 39(2), 1538-1545.

Emrouznejad, A., \& Yang, G. L. (2018). A survey and analysis of the first 40 years of scholarly literature in DEA: 1978-2016. Socio-Economic Planning Sciences, 61, 4-8. https://doi.org/10.1016/j.seps.2017.01.008

Espitia-Escuer, M., \& García-Cebrián, L. I. (2010). Measurement of the efficiency of football teams in the Champions League. Managerial and Decision Economics, 31(6), 373-386.

https://doi.org/10.1002/mde.1491

Espitia Escuer, M. A., \& García Cebrián, L. I. (2019). Procedure for Implementing Data Envelopment Analysis as a Methodology for Measuring Efficiency. Application to Football Teams. Application to Football Teams (June 27, 2019).

Färe, R., Grosskopf, S., Karagiannis, G., \& Margaritis, D. (2017). Data envelopment analysis and its related linear programming models. Annals of Operations Research, 250(1), 37-43. https://doi.org/10.1007/s10479-015-2042-y

Ferraz, D., Moralles, H. F., Campoli, J. S., Oliveira, F. C. R. D., \& Rebelatto, D. A. D. N. (2018). Complexidade Econômica e Desenvolvimento Humano: uma análise a partir do DEA. Gestão \& Produção, 25(4), 839-853. http://dx.doi.org/10.1590/0104-530x3925-18

Ferreira, C. M. D., Gomes, A. P. G. (2009). Introdução à análise envoltória de dados: teoria, modelos e aplicações. UFV.

Freitas, M. M. D., Farias, R. A. S., \& Flach, L. (2017). Efficiency determinants in Brazilian football clubs. Brazilian Business Review, 1-23. 
Glauner, F. (2018). Innovation, business models, and catastrophe: Reframing the mental model for innovation management. In Innovation Management and Corporate Social Responsibility (pp. 133-157). Springer, Cham. https://doi.org/10.1007/978-3-319-93629-1_7

Greene, W. H. (2008). The econometric approach to efficiency analysis. The measurement of productive efficiency and productivity growth, 1(1), 92-250.

Guzmán, I. (2006). Measuring efficiency and sustainable growth in Spanish football teams. European sport management quarterly, 6(3), 267-287. https://doi.org/10.1080/16184740601095040

Hamidi, M., Sajadi, H., \& Soleimani-Damaneh, J. (2011). Evaluating the performance of Iranian football teams utilizing linear programming. American Journal of Operations Research, 1(02), 65. https://doi.org/10.4236/ajor.2011.12010

Itaú, J. (2019, December 17). Análise econômico-financeira dos clubes de futebol brasileiros [Web page]. Retrieved from https://www.itau.com.br/_arquivosestaticos/itauBBA

Joro, T., \& Korhonen, P. (2015). Extension of data envelopment analysis with preference information. Springer. https://doi.org/10.1007/978-1-4899-7528-7

Kern, A., Schwarzmann, M., \& Wiedenegger, A. (2012). Measuring the efficiency of English Premier League football: A two-stage data envelopment analysis approach. Sport, Business and Management: an International Journal, 2(3), 177-195. https://doi.org/10.1108/20426781211261502

Levene, H. (1960). Levene test for equality of variances. Contributions to probability and statistics, 278-292.

Li, Y., Lei, X., Dai, Q., \& Liang, L. (2015). Performance evaluation of participating nations at the 2012 London Summer Olympics by a two-stage data envelopment analysis. European Journal of Operational Research, 243(3), 964-973. https://doi.org/10.1016/j.ejor.2014.12.032

Liu, W., Sharp, J., \& Wu, Z. (2006). Preference, production and performance in data envelopment analysis. Annals of Operations Research, 145(1), 105-127. https://doi.org/10.1007/s10479006-0042-7

Marotz, D. P., Marquezan, L. H. F., \& Diehl, C. A. (2020). Clubes de futebol: relações entre investimento, desempenho e adesão ao PROFUT. Revista Contemporânea de Contabilidade, 17(43), 3-18. https://doi.org/10.5007/2175-8069.2020v17n43p3

Meza, L. A., Biondi Neto, L., Mello, J. C. C. B. S., \& Gomes, E. G. (2005). ISYDS-Integrated System for Decision Support (SIAD-Sistema Integrado de Apoio à Decisão): a software package for data envelopment analysis model. Pesquisa Operacional, 25(3), 493-503. http://dx.doi.org/10.1590/s0101-74382005000300011

McDonald, J. (2009). Using least squares and tobit in second stage DEA efficiency analyses. European journal of operational research, 197(2), 792-798. https://doi.org/10.1016/j.ejor.2008.07.039

Mixon Jr, J. W., \& Smith, R. J. (2006). Teaching undergraduate econometrics with GRETL. Journal of Applied Econometrics, 21(7), 1103-1107. https://doi.org/10.1002/jae.927

Nascimento, J. C. H. B., Nossa, V., Bernardes, J. R., \& de Sousa, W. D. (2015). A eficiência dos maiores clubes de futebol brasileiros: evidências de uma análise longitudinal no período de 2006 a 2011. Contabilidade Vista \& Revista, 26(2), 137-161. 
Pereira, A. G. C., Júnior, A. C. B., Kronbauer, C. A., \& Abrantes, L. A. (2015). Eficiência técnica e desempenho econômico-financeiro dos clubes de futebol brasileiros. Revista Reuna, 20(2), 115-138.

Périco, A. E., Santana, N. B., \& Rebelatto, D. A. D. N. (2017). Eficiência dos aeroportos internacionais brasileiros: uma análise envoltória de dados com bootstrap. Gestão \& Produção, 24(2), 370381. http://dx.doi.org/10.1590/0104-530x1810-15

Pestana Barros, C., Assaf, A., \& Sá-Earp, F. (2010). Brazilian football league technical efficiency: a Simar and Wilson approach. Journal of Sports Economics, 11(6), 641-651. https://doi.org/10.1177/1527002509357530

Plumley, D., Wilson, R., \& Ramchandani, G. (2017). Towards a model for measuring holistic performance of professional Football clubs. Soccer \& Society, 18(1), 16-29. https://doi.org/10.1080/14660970.2014.980737

Pyatunin, A. V., Vishnyakova, A. B., Sherstneva, N. L., Mironova, S. P., Dneprov, S. A., \& Grabozdin, Y. P. (2016). The Economic Efficiency of European Football Clubs--Data Envelopment Analysis (DEA) Approach. International Journal of Environmental and Science Education, 11(15), 7515-7534.

Roll, Y., Cook, W. D., \& Golany, B. (1991). Controlling factor weights in data envelopment analysis. IIE transactions, 23(1), 2-9. https://doi.org/10.1080/07408179108963835

Rashidi, K., \& Cullinane, K. (2019). Evaluating the sustainability of national logistics performance using Data Envelopment Analysis. Transport Policy, 74, 35-46. https://doi.org/10.1016/j.tranpol.2018.11.014

Shih, C. P., \& Putri, D. U. (2018). Measuring the Effect of Team Characteristics, Team Effectiveness on Organizational Performance, Organizational Survival and Competitiveness: A Case Study of an Indonesian NGO, Yayasan Cinta Anak Bangsa by using PLS Method. In Operations and Service Management: Concepts, Methodologies, Tools, and Applications (pp. 1623-1636). IGI Global. https://doi.org/10.4018/978-1-5225-3909-4.ch073

Thanassoulis, E., Portela, M. C., \& Allen, R. (2004). Incorporating value judgments in DEA. In Handbook on data envelopment analysis (pp. 99-138). Springer, Boston, MA. https://doi.org/10.1007/14020-7798-X_4

Tran, T. H., Mao, Y., Nathanail, P., Siebers, P. O., \& Robinson, D. (2019). Integrating slacks-based measure of efficiency and super-efficiency in data envelopment analysis. Omega, 85, 156-165. https://doi.org/10.1016/j.omega.2018.06.008

Valério, R. P., \& Angulo-Meza, L. I. D. I. A. (2013). A data envelopment analysis evaluation and financial resources reallocation for Brazilian olympic sports. WSEAS Transactions on Systems, 12(12), 627-636.

Zhou, H., Yang, Y., Chen, Y., \& Zhu, J. (2018). Data envelopment analysis application in sustainability: The origins, development and future directions. European Journal of Operational Research, 264(1), 1-16. https://doi.org/10.1016/j.ejor.2017.06.023

Zhu, J. (2014). Quantitative models for performance evaluation and benchmarking: data envelopment analysis with spreadsheets (Vol. 213). Springer. https://doi.org/10.1007/978-3-319-06647-9 